\title{
$B^{0}-\bar{B}^{0}$ MIXING
}

Written March 2000 and revised March 2002 by O. Schneider (University of Lausanne).

\section{Formalism in quantum mechanics}

There are two neutral $B^{0}-\bar{B}^{0}$ meson systems, $B_{d}^{0}-\bar{B}_{d}^{0}$ and $B_{s}^{0}-\bar{B}_{s}^{0}$ (generically denoted $B_{q}^{0}-\bar{B}_{q}^{0}, q=s, d$ ), which exhibit the phenomenon of particle-antiparticle mixing [1]. Such a system is produced in one of its two possible states of well-defined flavor: $\left|B^{0}\right\rangle(\bar{b} q)$ or $\left|\bar{B}^{0}\right\rangle(b \bar{q})$. Due to flavor-changing interactions, this initial state evolves into a time-dependent quantum superposition of the two flavor states, $a(t)\left|B^{0}\right\rangle+b(t)\left|\bar{B}^{0}\right\rangle$, satisfying the equation

$$
i \frac{\partial}{\partial t}\left(\begin{array}{l}
a(t) \\
b(t)
\end{array}\right)=\left(\mathbf{M}-\frac{i}{2} \boldsymbol{\Gamma}\right)\left(\begin{array}{l}
a(t) \\
b(t)
\end{array}\right),
$$

where $\mathbf{M}$ and $\boldsymbol{\Gamma}$, known as the mass and decay matrices, describe the dispersive and absorptive parts of $B^{0}-\bar{B}^{0}$ mixing. These matrices are hermitian, and $C P T$ invariance requires $M_{11}=M_{22} \equiv M$ and $\Gamma_{11}=\Gamma_{22} \equiv \Gamma$

The two eigenstates of the effective Hamiltonian matrix $\left(\mathbf{M}-\frac{i}{2} \boldsymbol{\Gamma}\right)$ are given by

$$
\left|B_{ \pm}\right\rangle=p\left|B^{0}\right\rangle \pm q\left|\bar{B}^{0}\right\rangle
$$

and correspond to the eigenvalues

$$
\lambda_{ \pm}=\left(M-\frac{i}{2} \Gamma\right) \pm \frac{q}{p}\left(M_{12}-\frac{i}{2} \Gamma_{12}\right)
$$

where

$$
\frac{q}{p}=\sqrt{\frac{M_{12}^{*}-\frac{i}{2} \Gamma_{12}^{*}}{M_{12}-\frac{i}{2} \Gamma_{12}}}
$$

We choose a convention where $\operatorname{Re}(q / p)>0$ and $C P\left|B^{0}\right\rangle=\left|\bar{B}^{0}\right\rangle$.

An alternative notation is

$$
\left|B_{ \pm}\right\rangle=\frac{(1+\epsilon)\left|B^{0}\right\rangle \pm(1-\epsilon)\left|\bar{B}^{0}\right\rangle}{\sqrt{2\left(1+|\epsilon|^{2}\right)}} \quad \text { with } \quad \frac{1-\epsilon}{1+\epsilon}=\frac{q}{p}
$$

The time dependence of these eigenstates of well-defined masses $M_{ \pm}=\operatorname{Re}\left(\lambda_{ \pm}\right)$and decay widths $\Gamma_{ \pm}=-2 \operatorname{Im}\left(\lambda_{ \pm}\right)$is 
given by the phases $e^{-i \lambda_{ \pm} t}=e^{-i M_{ \pm} t} e^{-\frac{1}{2} \Gamma_{ \pm} t}$ : the evolution of a pure $\left|B^{0}\right\rangle$ or $\left|\bar{B}^{0}\right\rangle$ state at $t=0$ is thus given by

$$
\begin{aligned}
& \left|B^{0}(t)\right\rangle=g_{+}(t)\left|B^{0}\right\rangle+\frac{q}{p} g_{-}(t)\left|\bar{B}^{0}\right\rangle, \\
& \left|\bar{B}^{0}(t)\right\rangle=g_{+}(t)\left|\bar{B}^{0}\right\rangle+\frac{p}{q} g_{-}(t)\left|B^{0}\right\rangle,
\end{aligned}
$$

where

$$
g_{ \pm}(t)=\frac{1}{2}\left(e^{-i \lambda_{+} t} \pm e^{-i \lambda_{-} t}\right) .
$$

This means that the flavor states remain unchanged $(+)$ or oscillate into each other $(-)$ with time-dependent probabilities proportional to

$$
\left|g_{ \pm}(t)\right|^{2}=\frac{e^{-\Gamma t}}{2}\left[\cosh \left(\frac{\Delta \Gamma}{2} t\right) \pm \cos (\Delta m t)\right]
$$

where

$$
\Delta m=\left|M_{+}-M_{-}\right|, \quad \Delta \Gamma=\left|\Gamma_{+}-\Gamma_{-}\right| .
$$

Time-integrated mixing probabilities are only well defined when considering decays to flavor-specific final states, i.e. final states $f$ such that the instantaneous decay amplitudes $A_{\bar{f}}=\left\langle\bar{f}|H| B^{0}\right\rangle$ and $\bar{A}_{f}=\left\langle f|H| \bar{B}^{0}\right\rangle$, where $H$ is the weak interaction Hamiltonian, are both zero. Due to mixing, a produced $B^{0}$ can decay to the final state $\bar{f}$ (mixed event) in addition to the final state $f$ (unmixed event). Restricting the sample to these two decay channels, the time-integrated mixing probability is given by

$$
\begin{aligned}
\chi_{f}^{B^{0} \rightarrow \bar{B}^{0}} & =\frac{\int_{0}^{\infty}\left|\left\langle\bar{f}|H| B^{0}(t)\right\rangle\right|^{2} d t}{\int_{0}^{\infty}\left|\left\langle\bar{f}|H| B^{0}(t)\right\rangle\right|^{2} d t+\int_{0}^{\infty}\left|\left\langle f|H| B^{0}(t)\right\rangle\right|^{2} d t} \\
& =\frac{\left|\xi_{f}\right|^{2}\left(x^{2}+y^{2}\right)}{\left|\xi_{f}\right|^{2}\left(x^{2}+y^{2}\right)+2+x^{2}-y^{2}},
\end{aligned}
$$

where we have defined $\xi_{f}=\frac{q}{p} \frac{\overline{A_{\bar{f}}}}{A_{f}}$ and

$$
x=\frac{\Delta m}{\Gamma}, \quad y=\frac{\Delta \Gamma}{2 \Gamma} .
$$

The mixing probability $\chi_{f}^{\bar{B}^{0} \rightarrow B^{0}}$ for the case of a produced $\bar{B}^{0}$ is obtained by replacing $\xi_{f}$ with $1 / \xi_{f}$ in Eq. (11). It 
is different from $\chi_{f}^{B^{0} \rightarrow \bar{B}^{0}}$ if $\left|\xi_{f}\right|^{2} \neq 1$, a condition reflecting non-invariance under the $C P$ transformation. $C P$ violation in decay amplitudes is discussed elsewhere [2] and we assume $\left|\bar{A}_{\bar{f}}\right|=\left|A_{f}\right|$ from now on. The deviation of $|q / p|^{2}$ from 1, namely the quantity

$$
1-\left|\frac{q}{p}\right|^{2}=\frac{4 \operatorname{Re}(\epsilon)}{1+|\epsilon|^{2}}+\mathcal{O}\left(\left(\frac{\operatorname{Re}(\epsilon)}{1+|\epsilon|^{2}}\right)^{2}\right),
$$

describes $C P$ violation in $B^{0}-\bar{B}^{0}$ mixing. As can be seen from Eq. (4), this can occur only if $M_{12} \neq 0, \Gamma_{12} \neq 0$ and if the phase difference between $M_{12}$ and $\Gamma_{12}$ is different from 0 or $\pi$.

In the absence of $C P$ violation, $|q / p|^{2}=1, \operatorname{Re}(\epsilon)=0$, the mass eigenstates are also $C P$ eigenstates,

$$
C P\left|B_{ \pm}\right\rangle= \pm\left|B_{ \pm}\right\rangle
$$

the phases $\varphi_{M_{12}}=\arg \left(M_{12}\right)$ and $\varphi_{\Gamma_{12}}=\arg \left(\Gamma_{12}\right)$ satisfy

$$
\sin \left(\varphi_{M_{12}}-\varphi_{\Gamma_{12}}\right)=0,
$$

the mass and decay width differences reduce to

$$
\Delta m=2\left|M_{12}\right|, \quad \Delta \Gamma=2\left|\Gamma_{12}\right|,
$$

and the time-integrated mixing probabilities $\chi_{f}^{B^{0} \rightarrow \bar{B}^{0}}$ and $\chi_{f}^{\bar{B}^{0} \rightarrow B^{0}}$ become both equal to

$$
\chi=\frac{x^{2}+y^{2}}{2\left(x^{2}+1\right)} .
$$

\section{Standard Model predictions and phenomenology}

In the Standard Model, the transitions $B_{q}^{0} \rightarrow \bar{B}_{q}^{0}$ and $\bar{B}_{q}^{0} \rightarrow B_{q}^{0}$ are due to the weak interaction. They are described, at the lowest order, by box diagrams involving two $W$ bosons and two up-type quarks (see Fig. 1), as is the case for $K^{0}-\bar{K}^{0}$ mixing. However, the long range interactions arising from intermediate virtual states are negligible for the neutral $B$ meson systems, because the large $B$ mass is off the region of hadronic resonances. The calculation of the dispersive and absorptive parts of the box diagrams yields the following predictions for the off-diagonal element of the mass and decay matrices [3], 

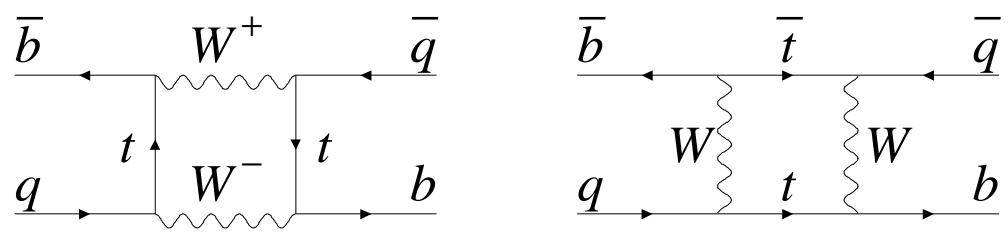

Figure 1: Dominant box diagrams for the $B_{q}^{0} \rightarrow \bar{B}_{q}^{0}$ transitions $(q=d$ or $s)$. Similar diagrams exist where one or both $t$ quarks are replaced with $c$ or $u$ quarks.

$$
\begin{aligned}
M_{12}= & -\frac{G_{F}^{2} m_{W}^{2} \eta_{B} m_{B_{q}} B_{B_{q}} f_{B_{q}}^{2}}{12 \pi^{2}} S_{0}\left(m_{t}^{2} / m_{W}^{2}\right)\left(V_{t q}^{*} V_{t b}\right)^{2} \\
\Gamma_{12}= & \frac{G_{F}^{2} m_{b}^{2} \eta_{B}^{\prime} m_{B_{q}} B_{B_{q}} f_{B_{q}}^{2}}{8 \pi} \\
& \times\left[\left(V_{t q}^{*} V_{t b}\right)^{2}+V_{t q}^{*} V_{t b} V_{c q}^{*} V_{c b} \mathcal{O}\left(\frac{m_{c}^{2}}{m_{b}^{2}}\right)\right. \\
& \left.\quad+\left(V_{c q}^{*} V_{c b}\right)^{2} \mathcal{O}\left(\frac{m_{c}^{4}}{m_{b}^{4}}\right)\right]
\end{aligned}
$$

where $G_{F}$ is the Fermi constant, $m_{W}$ the $W$ boson mass, $m_{i}$ the mass of quark $i$, and $m_{B_{q}}=M, f_{B_{q}}$ and $B_{B_{q}}$ are the $B_{q}^{0}$ mass, weak decay constant and bag parameter, respectively. The known function $S_{0}\left(x_{t}\right)$ can be approximated very well with $0.784 x_{t}^{0.76}$ [4] and $V_{i j}$ are the elements of the CKM matrix [5]. The QCD corrections $\eta_{B}$ and $\eta_{B}^{\prime}$ are of order unity. The only non negligible contributions to $M_{12}$ are from box diagrams involving two top quarks. The phases of $M_{12}$ and $\Gamma_{12}$ satisfy

$$
\varphi_{M_{12}}-\varphi_{\Gamma_{12}}=\pi+\mathcal{O}\left(\frac{m_{c}^{2}}{m_{b}^{2}}\right)
$$

implying that the mass eigenstates have mass and width differences of opposite signs. This means that, like in the $K^{0}-\bar{K}^{0}$ system, the "heavy" state with mass $M_{\text {heavy }}=\max \left(M_{+}, M_{-}\right)$ has a smaller decay width than that of the "light" state with mass $M_{\text {light }}=\min \left(M_{+}, M_{-}\right)$. We thus redefine

$$
\Delta m=M_{\text {heavy }}-M_{\text {light }}, \quad \Delta \Gamma=\Gamma_{\text {light }}-\Gamma_{\text {heavy }},
$$

where $\Delta m$ is positive by definition and $\Delta \Gamma$ is expected to be positive in the Standard Model. 
Furthermore, the quantity

$$
\left|\frac{\Gamma_{12}}{M_{12}}\right| \simeq \frac{3 \pi}{2} \frac{m_{b}^{2}}{m_{W}^{2}} \frac{1}{S_{0}\left(m_{t}^{2} / m_{W}^{2}\right)} \sim \mathcal{O}\left(\frac{m_{b}^{2}}{m_{t}^{2}}\right)
$$

is small, and a power expansion of $|q / p|^{2}$ yields

$$
\left|\frac{q}{p}\right|^{2}=1+\left|\frac{\Gamma_{12}}{M_{12}}\right| \sin \left(\varphi_{M_{12}}-\varphi_{\Gamma_{12}}\right)+\mathcal{O}\left(\left|\frac{\Gamma_{12}}{M_{12}}\right|^{2}\right) .
$$

Therefore, considering both Eqs. (20) and (22), the CPviolating parameter

$$
1-\left|\frac{q}{p}\right|^{2} \simeq \operatorname{Im}\left(\frac{\Gamma_{12}}{M_{12}}\right)
$$

is expected to be very small: $\sim \mathcal{O}\left(10^{-3}\right)$ for the $B_{d}^{0}-\bar{B}_{d}^{0}$ system and $\lesssim \mathcal{O}\left(10^{-4}\right)$ for the $B_{s}^{0}-\bar{B}_{s}^{0}$ system [6].

In the approximation of negligible $C P$ violation in mixing, the ratio $\Delta \Gamma / \Delta m$ is equal to the small quantity $\left|\Gamma_{12} / M_{12}\right|$ of Eq. (22); it is hence independent of CKM matrix elements, i.e. the same for the $B_{d}^{0}-\bar{B}_{d}^{0}$ and $B_{s}^{0}-\bar{B}_{s}^{0}$ systems. It can be calculated with lattice QCD techniques; typical results are $\sim 5 \times 10^{-3}$ with quoted uncertainties of $\sim 30 \%$. Given the current experimental knowledge (discussed below) on the mixing parameter $x$,

$$
\left\{\begin{array}{ll}
x_{d}=0.755 \pm 0.015 & \left(B_{d}^{0}-\bar{B}_{d}^{0} \text { system }\right) \\
x_{s}>19.0 \text { at } 95 \% \mathrm{CL} & \left(B_{s}^{0}-\bar{B}_{s}^{0} \text { system }\right)
\end{array},\right.
$$

the Standard Model thus predicts that $\Delta \Gamma / \Gamma$ is very small for the $B_{d}^{0}-\bar{B}_{d}^{0}$ system (below $1 \%$ ), but considerably larger for the $B_{s}^{0}-\bar{B}_{s}^{0}$ system $(\sim 10 \%)$. This width difference is caused by the existence of final states to which both the $B_{q}^{0}$ and $\bar{B}_{q}^{0}$ mesons can decay. Such decays involve $b \rightarrow c \bar{c} q$ quarklevel transitions, which are Cabibbo-suppressed if $q=d$ and Cabibbo-allowed if $q=s$. If the final states common to $B_{s}^{0}$ and $\bar{B}_{s}^{0}$ are predominantly $C P$-even as discussed in Ref. 7 , then the $B_{s}^{0}-\bar{B}_{s}^{0}$ mass eigenstate with the largest decay width corresponds to the $C P$-even eigenstate. Taking Eq. (21) into account, one thus expects $\Gamma_{\text {light }}=\Gamma_{+}$and

$$
\Delta m_{s}=M_{-}-M_{+}>0, \quad \Delta \Gamma_{s}=\Gamma_{+}-\Gamma_{-}>0 .
$$




\section{Experimental issues and methods for oscillation anal- yses}

Time-integrated measurements of $B^{0}-\bar{B}^{0}$ mixing were published for the first time in 1987 by UA1 [8] and ARGUS [9], and since then by many other experiments. These measurements are typically based on counting same-sign and opposite-sign lepton pairs from the semileptonic decay of the produced $b \bar{b}$ pairs. Such analyses cannot easily separate the contributions from the different $b$-hadron species, therefore the clean environment of $\Upsilon(4 S)$ machines (where only $B_{d}^{0}$ and charged $B_{u}$ mesons are produced) is in principle best suited to measure $\chi_{d}$.

However, better sensitivity is obtained from time-dependent analyses aimed at the direct measurement of the oscillation frequencies $\Delta m_{d}$ and $\Delta m_{s}$, from the proper time distributions of $B_{d}^{0}$ or $B_{s}^{0}$ candidates identified through their decay in (mostly) flavor-specific modes and suitably tagged as mixed or unmixed. (This is particularly true for the $B_{s}^{0}-\bar{B}_{s}^{0}$ system where the large value of $x_{s}$ implies maximal mixing, i.e. $\chi_{s} \simeq 1 / 2$.) In such analyses the $B_{d}^{0}$ or $B_{s}^{0}$ mesons are either fully reconstructed, partially reconstructed from a charm meson, selected from a lepton with the characteristics of a $b \rightarrow \ell^{-}$decay, or selected from a reconstructed displaced vertex. At high-energy colliders (LEP, SLC, Tevatron), the proper time $t=\frac{m_{B}}{p} L$ is measured from the distance $L$ between the production vertex and the $B$ decay vertex, and from an estimate of the $B$ momentum $p$. At asymmetric $B$ factories (KEKB, PEP-II), producing $e^{+} e^{-} \rightarrow \Upsilon(4 S) \rightarrow B_{d}^{0} \bar{B}_{d}^{0}$ events with a boost $\beta \gamma(=0.425$, $0.55)$, the proper time difference between the two $B$ candidates is estimated as $\Delta t \simeq \frac{\Delta z}{\beta \gamma c}$, where $\Delta z$ is the spatial separation between the two $B$ decay vertices along the boost direction. In all cases, the good resolution needed on the vertex positions is obtained with silicon detectors.

The statistical significance $\mathcal{S}$ of a $B_{d}^{0}$ or $B_{s}^{0}$ oscillation signal can be approximated as $[10]$

$$
\mathcal{S} \approx \sqrt{N / 2} f_{\text {sig }}(1-2 \eta) e^{-\left(\Delta m \sigma_{t}\right)^{2} / 2}
$$

where $N$ and $f_{\text {sig }}$ are the number of candidates and the fraction of signal in the selected sample, $\eta$ is the total mistag probability, 
and $\sigma_{t}$ is the resolution on proper time (or proper time difference). The quantity $\mathcal{S}$ decreases very quickly as $\Delta m$ increases; this dependence is controlled by $\sigma_{t}$, which is therefore a critical parameter for $\Delta m_{s}$ analyses. At high-energy colliders, the proper time resolution $\sigma_{t} \sim \frac{m_{B}}{\langle p\rangle} \sigma_{L} \oplus t \frac{\sigma_{p}}{p}$ includes a constant contribution due to the decay length resolution $\sigma_{L}$ (typically 0.05- $0.3 \mathrm{ps}$ ), and a term due to the relative momentum resolution $\sigma_{p} / p$ (typically $10-20 \%$ for partially reconstructed decays) which increases with proper time. At $B$ factories, the $B$ momentum is reconstructed and/or estimated from the beam energy constraint, and the term due to the spatial resolution dominates (typically $1-1.5$ ps because of the much smaller $B$ boost).

In order to tag a $B$ candidate as mixed or unmixed, it is necessary to determine its flavor both in the initial state and in the final state. The initial and final state mistag probabilities, $\eta_{i}$ and $\eta_{f}$, degrade $\mathcal{S}$ by a total factor $(1-2 \eta)=\left(1-2 \eta_{i}\right)\left(1-2 \eta_{f}\right)$. In lepton-based analyses, the final state is tagged by the charge of the lepton from $b \rightarrow \ell^{-}$decays; the biggest contribution to $\eta_{f}$ is then due to $\bar{b} \rightarrow \bar{c} \rightarrow \ell^{-}$decays. Alternatively, the charge of a reconstructed charm meson $\left(D^{*-}\right.$ from $B_{d}^{0}$ or $D_{s}^{-}$from $\left.B_{s}^{0}\right)$, or that of a kaon thought to come from a $b \rightarrow c \rightarrow s$ decay [11], can be used. For fully inclusive analyses based on topological vertexing, final state tagging techniques include jet charge [12] and charge dipole $[13,14]$ methods.

At high-energy colliders, the methods to tag the initial state (i.e. the state at production), can be divided in two groups: the ones that tag the initial charge of the $\bar{b}$ quark contained in the $B$ candidate itself (same-side tag), and the ones that tag the initial charge of the other $b$ quark produced in the event (opposite-side tag). On the same side, the charge of a track from the primary vertex is correlated with the production state of the $B$ if that track is a decay product of a $B^{* *}$ state or the first particle in the fragmentation chain $[15,16]$. Jet- and vertex-charge techniques work on both sides and on the opposite side, respectively. Finally, the charge of a lepton from $b \rightarrow \ell^{-}$ or of a kaon from $b \rightarrow c \rightarrow s$ can be used as opposite side tags, keeping in mind that their performance is degraded due to integrated mixing. At SLC, the beam polarization produced 
a sizeable forward-backward asymmetry in the $Z \rightarrow b \bar{b}$ decays and provided another very interesting and effective initial state tag based on the polar angle of the $B$ candidate [11]. Initial state tags have also been combined to reach $\eta_{i} \sim 26 \%$ at LEP $[16,17]$, or even $22 \%$ at SLD [13] with full efficiency. The equivalent figure at CDF (Tevatron Run I) is 40\% [18].

At $B$ factories, the flavor of a $B_{d}^{0}$ meson at production cannot be determined, since the two neutral $B$ mesons produced in a $\Upsilon(4 S)$ decay evolve in a coherent $P$-wave state where they keep opposite flavors at any time. However, as soon as one of them decays, the other follows a time-evolution given by Eqs. (6) or (7), where $t$ is replaced with $\Delta t$. Hence, the "initial state" tag of a $B$ can be taken as the final state tag of the other $B$. Effective mistag probabilities of $\eta_{i} \sim 24 \%$ for full efficiency (corresponding to effective tagging efficiencies of $\sim 27 \%$ for perfect tagging) are achieved by BABAR and Belle [19], using different techniques including $b \rightarrow \ell^{-}$and $b \rightarrow c \rightarrow s$ tags. It is interesting to note that, in this case, mixing of this other $B$ (i.e. the coherent mixing occurring before the first $B$ decay) does not contribute to the mistag probability.

In the absence of experimental evidence for a width difference, oscillation analyses typically neglect $\Delta \Gamma$ and describe the data with the physics functions $\Gamma e^{-\Gamma t}(1 \pm \cos (\Delta m t)) / 2$ (highenergy colliders) or $\Gamma e^{-\Gamma|\Delta t|}(1 \pm \cos (\Delta m \Delta t)) / 4$ (asymmetric $\Upsilon(4 S)$ machines). As can be seen from Eq. (9), a non-zero value of $\Delta \Gamma$ would effectively reduce the oscillation amplitude with a small time-dependent factor that would be very difficult to distinguish from time resolution effects. Whereas measurements of $\Delta m_{d}$ are usually extracted from the data using a maximum likelihood fit, no significant $B_{s}^{0}-\bar{B}_{s}^{0}$ oscillations have been seen so far. To extract information useful to set lower limits on $\Delta m_{s}, B_{s}^{0}$ analyses follow a method [10] in which a $B_{s}^{0}$ oscillation amplitude $\mathcal{A}$ is measured as a function of a fixed test value of $\Delta m_{s}$, using a maximum likelihood fit based on the functions $\Gamma_{s} e^{-\Gamma_{s} t}\left(1 \pm \mathcal{A} \cos \left(\Delta m_{s} t\right)\right) / 2$. To a very good approximation, the statistical uncertainty on $\mathcal{A}$ is Gaussian and equal to $1 / \mathcal{S}$ [10]. If $\Delta m_{s}=\Delta m_{s}^{\text {true }}$, one expects $\mathcal{A}=1$ within the total uncertainty $\sigma_{\mathcal{A}}$; however, if $\Delta m_{s}$ is (far) below its true 
value, a measurement consistent with $\mathcal{A}=0$ is expected. A value of $\Delta m_{s}$ can be excluded at $95 \% \mathrm{CL}$ if $\mathcal{A}+1.645 \sigma_{\mathcal{A}} \leq 1$. If $\Delta m_{s}^{\text {true }}$ is very large, one expects $\mathcal{A}=0$, and all values of $\Delta m_{s}$ such that $1.645 \sigma_{\mathcal{A}}\left(\Delta m_{s}\right)<1$ are expected to be excluded at $95 \% \mathrm{CL}$. Because of the proper time resolution, the quantity $\sigma_{\mathcal{A}}\left(\Delta m_{s}\right)$ is an increasing function of $\Delta m_{s}$ and one therefore expects to be able to exclude individual $\Delta m_{s}$ values up to $\Delta m_{s}^{\text {sens }}$, where $\Delta m_{s}^{\text {sens }}$, called here the sensitivity of the analysis, is defined by $1.645 \sigma_{\mathcal{A}}\left(\Delta m_{s}^{\text {sens }}\right)=1$.

\section{$B_{d}^{0}$ mixing studies}

Many $B_{d}^{0}-\bar{B}_{d}^{0}$ oscillations analyses have been performed by the ALEPH [12,20], BABAR [21], Belle [22,23], CDF [15,24], DELPHI [14,25], L3 [26], OPAL [27] and SLD [11] collaborations. Although a variety of different techniques have been used, the individual $\Delta m_{d}$ results obtained at high-energy colliders have remarkably similar precision. Their average is compatible with the recent and more precise measurements from asymmetric $B$ factories. The systematic uncertainties are not negligible; they are often dominated by sample composition, mistag probability, or $b$-hadron lifetime contributions. Before being combined, the measurements are adjusted on the basis of a common set of input values, including the $b$-hadron lifetimes and fractions published in this Review. Some measurements are statistically correlated. Systematic correlations arise both from common physics sources (fragmentation fractions, lifetimes, branching ratios of $b$ hadrons), and from purely experimental or algorithmic effects (efficiency, resolution, tagging, background description). Combining all published measurements [15,20-22,25-27] and accounting for all identified correlations as described in Ref. 28 yields $\Delta m_{d}=0.489 \pm 0.005$ (stat) \pm 0.007 (syst) $\mathrm{ps}^{-1}$.

On the other hand, ARGUS and CLEO have published time-integrated measurements [29-31], which average to $\chi_{d}=$ $0.182 \pm 0.015$. Following Ref. 31, the width difference $\Delta \Gamma_{d}$ could in principle be extracted from the measured value of $\Gamma_{d}$ and the above averages for $\Delta m_{d}$ and $\chi_{d}$ (see Eqs. (12) and (17)), provided that $\Delta \Gamma_{d}$ has a negligible impact on the $\Delta m_{d}$ measurements. However, a stronger constraint, $\Delta \Gamma_{d} / \Gamma_{d}<20 \%$ 
at $90 \% \mathrm{CL}$, has been obtained by DELPHI from a direct timedependent study [14]. Assuming $\Delta \Gamma_{d}=0$ and no CP violation in mixing, and using the measured $B_{d}^{0}$ lifetime, the $\Delta m_{d}$ and $\chi_{d}$ results are combined to yield the world average

$$
\Delta m_{d}=0.489 \pm 0.008 \mathrm{ps}^{-1}
$$

or, equivalently,

$$
\chi_{d}=0.181 \pm 0.004 .
$$

Evidence for $C P$ violation in $B_{d}^{0}$ mixing has been searched for, both with flavor-specific and inclusive $B_{d}^{0}$ decays, in samples where the initial flavor state is tagged. In the case of semileptonic (or other flavor-specific) decays, where the final state tag is also available, the following asymmetry

$$
\begin{aligned}
& \frac{N\left(\bar{B}_{d}^{0}(t) \rightarrow \ell^{+} \nu_{\ell} X\right)-N\left(B_{d}^{0}(t) \rightarrow \ell^{-} \bar{\nu}_{\ell} X\right)}{N\left(\bar{B}_{d}^{0}(t) \rightarrow \ell^{+} \nu_{\ell} X\right)+N\left(B_{d}^{0}(t) \rightarrow \ell^{-} \bar{\nu}_{\ell} X\right)} \\
& =a_{C P} \simeq 1-|q / p|_{d}^{2} \simeq \frac{4 \operatorname{Re}\left(\epsilon_{d}\right)}{1+\left|\epsilon_{d}\right|^{2}}
\end{aligned}
$$

has been measured, either in time-integrated analyses at CLEO [30-32] and CDF [33], or in time-dependent analyses at LEP [34-36] and BABAR [37]. In the inclusive case, also investigated at LEP $[35,36,38]$, no final state tag is used, and the asymmetry [39]

$$
\begin{aligned}
& \frac{N\left(B_{d}^{0}(t) \rightarrow \text { all }\right)-N\left(\bar{B}_{d}^{0}(t) \rightarrow \text { all }\right)}{N\left(B_{d}^{0}(t) \rightarrow \text { all }\right)+N\left(\bar{B}_{d}^{0}(t) \rightarrow \text { all }\right)} \\
& \simeq a_{C P}\left[\frac{x_{d}}{2} \sin \left(\Delta m_{d} t\right)-\sin ^{2}\left(\frac{\Delta m_{d} t}{2}\right)\right]
\end{aligned}
$$

must be measured as a function of the proper time to extract information on $C P$ violation. In all cases asymmetries compatible with zero have been found, with a precision limited by the available statistics. A simple average of all published and preliminary results [30-38] neglecting small possible statistical correlations and assuming half of the systematics to be correlated between measurements performed at the same energy, is $a_{C P}=-0.002 \pm 0.009$ (stat) \pm 0.008 (syst), a result which does not yet constrain the Standard Model. 
The $\Delta m_{d}$ result of Eq. (28) provides an estimate of $\left|M_{12}\right|$ and can be used, together with Eqs. (16) and (18), to extract the magnitude of the CKM matrix element $V_{t d}$ within the Standard Model [40]. The main experimental uncertainties on the resulting estimate of $\left|V_{t d}\right|$ come from $m_{t}$ and $\Delta m_{d}$; however, the extraction is at present completely dominated by the uncertainty on the hadronic matrix element $f_{B_{d}} \sqrt{B_{B_{d}}}=230 \pm 40 \mathrm{MeV}$ obtained from lattice QCD calculations [41].

\section{$B_{s}^{0}$ mixing studies}

$B_{s}^{0}-\bar{B}_{s}^{0}$ oscillations have been the subject of many studies from ALEPH [16,42], CDF [43], DELPHI [14,17,44,45], OPAL [46] and SLD [13,47]. No oscillation signal has been found so far. The most sensitive analyses appear to be the ones based on inclusive lepton samples at LEP. Because of their better proper time resolution, the small data samples analyzed inclusively at SLD, as well as the few fully reconstructed $B_{s}$ decays at LEP, turn out to be also very useful to explore the high $\Delta m_{s}$ region.

All results are limited by the available statistics. They can easily be combined, since all experiments provide measurements of the $B_{s}^{0}$ oscillation amplitude. The latter are averaged using the procedure of Ref. 28 to yield the combined amplitudes $\mathcal{A}$ shown in Fig. 2 as a function of $\Delta m_{s}$. The individual results have been adjusted to common physics inputs, and all known correlations have been accounted for; the sensitivities of the inclusive analyses, which depend directly through Eq. (27) on the assumed fraction $f_{s}$ of $B_{s}^{0}$ mesons in an unbiased sample of weakly-decaying $b$ hadrons, have also been rescaled to a common preliminary average of $f_{s}=0.097 \pm 0.011$. The combined sensitivity for 95\% CL exclusion of $\Delta m_{s}$ values is found to be $19.3 \mathrm{ps}^{-1}$. All values of $\Delta m_{s}$ below $14.9 \mathrm{ps}^{-1}$ are excluded at $95 \% \mathrm{CL}$. The values between 14.9 and $22.4 \mathrm{ps}^{-1}$ cannot be excluded, because the data is compatible with a signal in this region. However, no deviation from $\mathcal{A}=0$ is seen in Fig. 2 that would indicate the observation of a signal.

Some $\Delta m_{s}$ analyses are still unpublished [13,14,42,45,47]. Using only published results, the combined $\Delta m_{s}$ result is

$$
\begin{gathered}
\Delta m_{s}>13.1 \mathrm{ps}^{-1} \quad \text { at } 95 \% \mathrm{CL}, \\
\text { June } 21,2002 \quad 10: 27
\end{gathered}
$$




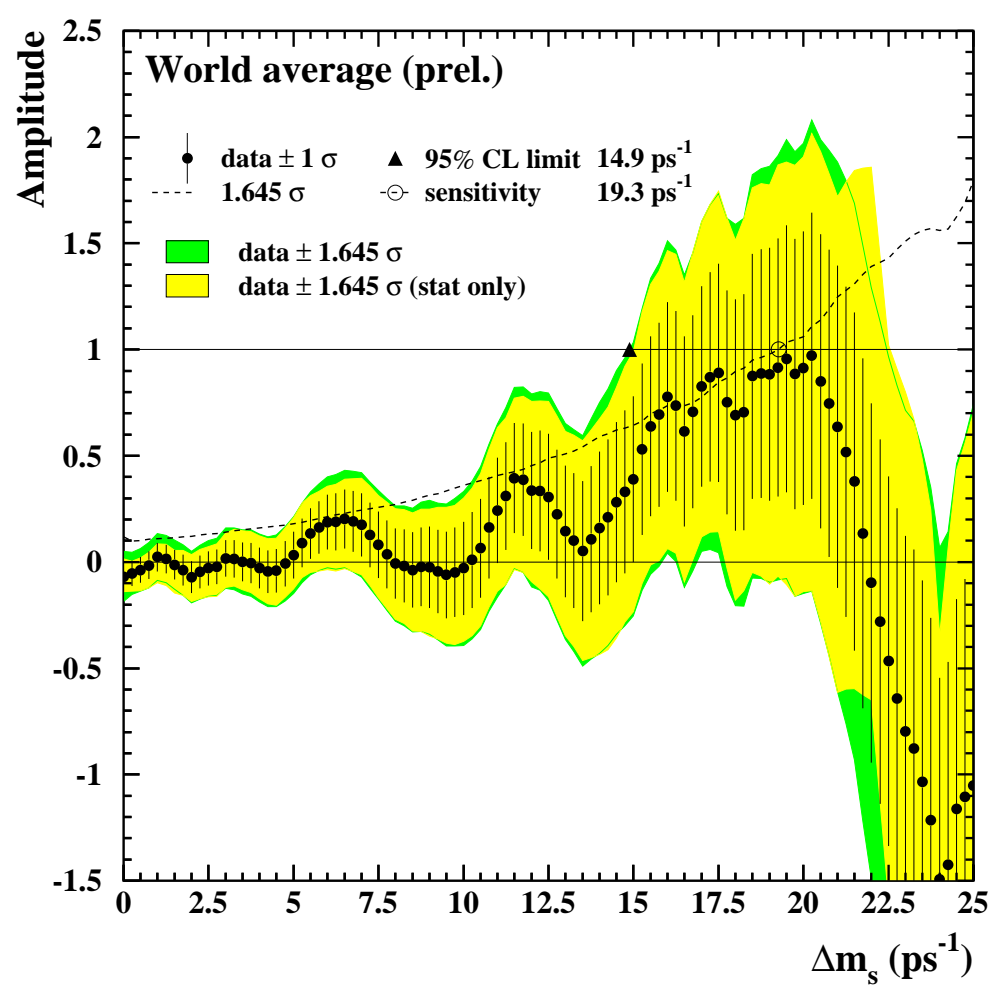

Figure 2: Combined measurements of the $B_{s}^{0}$ oscillation amplitude as a function of $\Delta m_{s}$, including all preliminary results available at the time of the winter 2002 conferences [48]. The measurements are dominated by statistical uncertainties. Neighboring points are statistically correlated.

with a sensitivity of $13.3 \mathrm{ps}^{-1}$.

The information on $\left|V_{t s}\right|$ obtained, in the framework of the Standard Model, from the combined amplitude spectrum is hampered by the hadronic uncertainty, as in the $B_{d}^{0}$ case. However, many uncertainties cancel in the frequency ratio

$$
\frac{\Delta m_{s}}{\Delta m_{d}}=\frac{m_{B_{s}}}{m_{B_{d}}} \xi^{2}\left|\frac{V_{t s}}{V_{t d}}\right|^{2},
$$

where $\xi=\left(f_{B_{s}} \sqrt{B_{B_{s}}}\right) /\left(f_{B_{d}} \sqrt{B_{B_{d}}}\right)=1.16 \pm 0.05$ is an $\mathrm{SU}(3)$ flavor-symmetry breaking factor obtained from lattice QCD calculations [41]. The CKM matrix can be constrained using the experimental results on $\Delta m_{d}, \Delta m_{s},\left|V_{u b} / V_{c b}\right|, \epsilon_{K}$ and $\sin (2 \beta)$ together with theoretical inputs and unitarity conditions $[40,49]$. Given all measurements other than $\Delta m_{d}$ and $\Delta m_{s}$, the constraint from our knowledge on the ratio $\Delta m_{d} / \Delta m_{s}$ is presently more effective in limiting the position of the apex of the CKM 
unitarity triangle than the one obtained from the $\Delta m_{d}$ measurements alone, due to the reduced hadronic uncertainty in Eq. (33). We note also that it would be difficult for the Standard Model to accommodate values of $\Delta m_{s}$ above $\sim 25 \mathrm{ps}^{-1}$ [49].

Information on $\Delta \Gamma_{s}$ can be obtained by studying the proper time distribution of untagged data samples enriched in $B_{s}^{0}$ mesons [50]. In the case of an inclusive $B_{s}^{0}$ selection [51] or a semileptonic $B_{s}^{0}$ decay selection $[17,52]$, both the short- and long-lived components are present, and the proper time distribution is a superposition of two exponentials with decay constants $\Gamma_{s} \pm \Delta \Gamma_{s} / 2$. In principle, this provides sensitivity to both $\Gamma_{s}$ and $\left(\Delta \Gamma_{s} / \Gamma_{s}\right)^{2}$. Ignoring $\Delta \Gamma_{s}$ and fitting for a single exponential leads to an estimate of $\Gamma_{s}$ with a relative bias proportional to $\left(\Delta \Gamma_{s} / \Gamma_{s}\right)^{2}$. An alternative approach, which is directly sensitive to first order in $\Delta \Gamma_{s} / \Gamma_{s}$, is to determine the lifetime of $B_{s}^{0}$ candidates decaying to $C P$ eigenstates; measurements exist for $B_{s}^{0} \rightarrow J / \psi \phi[53]$ and $B_{s}^{0} \rightarrow D_{s}^{(*)+} D_{s}^{(*)-}[54]$, which are mostly $C P$-even states [7]. An estimate of $\Delta \Gamma_{s} / \Gamma_{s}$ has also been obtained directly from a measurement of the $B_{s}^{0} \rightarrow D_{s}^{(*)+} D_{s}^{(*)-}$ branching ratio [54], under the assumption that these decays practically account for all the $C P$-even final states.

Present data is not precise enough to efficiently constrain both $\Gamma_{s}$ and $\Delta \Gamma_{s} / \Gamma_{s}$; since the $B_{s}^{0}$ and $B_{d}^{0}$ lifetimes are predicted to be equal within less than a percent [55], an expectation compatible with the current experimental data [56], the constraint $\Gamma_{s}=\Gamma_{d}$ can also be used to improve the extraction of $\Delta \Gamma_{s} / \Gamma_{s}$. Applying the combination procedure of Ref. 28 on the published results $[17,52-54,57]$ yields

$$
\Delta \Gamma_{s} / \Gamma_{s}<0.52 \quad \text { at } 95 \% \mathrm{CL}
$$

without external constraint, or

$$
\Delta \Gamma_{s} / \Gamma_{s}<0.31 \quad \text { at } 95 \% \mathrm{CL}
$$

when constraining $1 / \Gamma_{s}$ to the measured $B_{d}^{0}$ lifetime. These results are not yet precise enough to test Standard Model predictions. 


\section{Average b-hadron mixing and b-hadron production frac- tions at high energy}

Let $f_{u}, f_{d}, f_{s}$ and $f_{\text {baryon }}$ be the $B_{u}, B_{d}^{0}, B_{s}^{0}$ and $b$ baryon fractions composing an unbiased sample of weaklydecaying $b$ hadrons produced in high-energy colliders. LEP experiments have measured $f_{s} \times \mathrm{BR}\left(B_{s}^{0} \rightarrow D_{s}^{-} \ell^{+} \nu_{\ell} X\right)$ [58], $\mathrm{BR}\left(b \rightarrow \Lambda_{b}^{0}\right) \times \mathrm{BR}\left(\Lambda_{b}^{0} \rightarrow \Lambda_{c}^{+} \ell^{-} \bar{\nu}_{\ell} X\right)[59]$ and $\mathrm{BR}\left(b \rightarrow \Xi_{b}^{-}\right) \times$ $\operatorname{BR}\left(\Xi_{b}^{-} \rightarrow \Xi^{-} \ell^{-} \bar{\nu}_{\ell} X\right)$ [60] from partially reconstructed final states including a lepton, $f_{\text {baryon }}$ from protons identified in $b$ events [61], and the production rate of charged $b$ hadrons [62]. The various $b$-hadron fractions have also been measured at CDF from electron-charm final states [63]. All the published results have been combined following the procedure and assumptions described in Ref. 28, to yield $f_{u}=f_{d}=(37.3 \pm 2.0) \%, f_{s}=$ $(13.9 \pm 3.8) \%$ and $f_{\text {baryon }}=(11.5 \pm 2.0) \%$ under the constraints

$$
f_{u}=f_{d} \quad \text { and } \quad f_{u}+f_{d}+f_{s}+f_{\text {baryon }}=1 .
$$

Time-integrated mixing analyses performed with lepton pairs from $b \bar{b}$ events produced at high-energy colliders measure the quantity

$$
\bar{\chi}=f_{d}^{\prime} \chi_{d}+f_{s}^{\prime} \chi_{s}
$$

where $f_{d}^{\prime}$ and $f_{s}^{\prime}$ are the fractions of $B_{d}^{0}$ and $B_{s}^{0}$ hadrons in a sample of semileptonic $b$-hadron decays. Assuming that all $b$ hadrons have the same semileptonic decay width implies $f_{q}^{\prime}=f_{q} /\left(\Gamma_{q} \tau_{b}\right)(q=s, d)$, where $\tau_{b}$ is the average $b$-hadron lifetime. Hence $\bar{\chi}$ measurements can be used to improve our knowledge on the fractions $f_{u}, f_{d}, f_{s}$ and $f_{\text {baryon. }}$

Combining the above estimates of these fractions with the average $\bar{\chi}=0.1184 \pm 0.0045$ (published in this Review), $\chi_{d}$ from Eq. (29) and $\chi_{s}=1 / 2$ yields, under the constraints of Eq. (36),

$$
\begin{array}{r}
f_{u}=f_{d}=(38.8 \pm 1.3) \%, \\
f_{s}=(10.6 \pm 1.3) \%, \\
f_{\text {baryon }}=(11.8 \pm 2.0) \%,
\end{array}
$$

showing that mixing information substantially reduces the uncertainty on $f_{s}$. These results and the averages quoted in 
Eqs. (28) and (29) for $\chi_{d}$ and $\Delta m_{d}$ have been obtained in a consistent way by the $B$ oscillations working group [28], taking into account the fact that many individual measurements of $\Delta m_{d}$ depend on the assumed values for the $b$-hadron fractions.

\section{Summary and prospects}

$B^{0}-\bar{B}^{0}$ mixing has been and still is a field of intense study. The mass difference in the $B_{d}^{0}-\bar{B}_{d}^{0}$ system is very well measured (with an accuracy of $1.7 \%$ ) but, despite an impressive theoretical effort, the hadronic uncertainty still limits the precision of the extracted estimate of $\left|V_{t d}\right|$. The mass difference in the $B_{s}^{0-}$ $\bar{B}_{s}^{0}$ system is much larger and still unmeasured. However, the current experimental lower limit on $\Delta m_{s}$ already provides, together with $\Delta m_{d}$, a significant constraint on the CKM matrix within the Standard Model. No strong experimental evidence exists yet for the rather large decay width difference expected in the $B_{s}^{0}-\bar{B}_{s}^{0}$ system. It is interesting to recall that the ratio $\Delta \Gamma_{s} / \Delta m_{s}$ does not depend on CKM matrix elements in the Standard Model (see Eq. (22)), and that a measurement of either $\Delta m_{s}$ or $\Delta \Gamma_{s}$ could be turned into a Standard Model prediction of the other one.

The LEP and SLD experiments have still not finalized all their $B_{s}^{0}$ oscillation analyses, but a first measurement of $\Delta m_{s}$ from data collected at the $Z$ pole is now very unlikely. In the near future, the most promising prospects for $B_{s}^{0}$ mixing are from Run II at the Tevatron, where both $\Delta m_{s}$ and $\Delta \Gamma_{s}$ are expected to be measured with fully reconstructed $B_{s}^{0}$ decays; for example, with $2 \mathrm{fb}^{-1}$ of data, CDF expects to observe $B_{s}^{0}$ oscillations for values of $\Delta m_{s}$ up to $\sim 40-50 \mathrm{ps}^{-1}$ (depending on event yields and signal-to-background ratios) [64], well above the current Standard Model prediction.

$C P$ violation in $B$ mixing, which has not been seen yet, as well as the phases involved in $B$ mixing, will be further investigated with the large statistics that will become available both at the $B$ factories and at the Tevatron.

$B$ mixing may not have delivered all its secrets yet, because it is one of the phenomena where new physics might very well reveal itself (for example new particles involved in the box 
diagrams). Theoretical calculations in lattice QCD are becoming more reliable and further progress in reducing hadronic uncertainties is expected. In the long term, a stringent check of the consistency, within the Standard Model, of the $B_{d}^{0}$ and $B_{s}^{0}$ mixing measurements with all other measured observables in $B$ physics (including $C P$ asymmetries in $B$ decays) will be possible, allowing to place limits on new physics or, better, discover new physics.

\section{References}

1. T.D. Lee and C.S. Wu, Ann. Rev. Nucl. Sci. 16, 511 (1966); I.I. Bigi and A.I. Sanda, " $C P$ violation," Cambridge, Cambridge Univ. Press, 2000;

G.C. Branco, L. Lavoura, and J.P. Silva, " $C P$ violation," Clarendon Press Oxford, 1999;

see also the review on $B^{0}-\bar{B}^{0}$ mixing by $\mathrm{H}$. Quinn in C. Caso et al., Eur. Phys. J. C3, 1 (1998).

2. See the review on $C P$ violation in $B$ decays by $\mathrm{H}$. Quinn and A. Sanda in this publication.

3. A.J. Buras, W. Slominski, and H. Steger, Nucl. Phys. B245, 369 (1984).

4. A.J. Buras and R. Fleischer, in "Heavy Flavours II," ed. A.J. Buras and M. Lindner, Singapore World Scientific (1998).

5. M. Kobayashi and K. Maskawa, Prog. Theor. Phys. 49, 652 (1973).

6. I.I. Bigi et al., in " $C P$ violation," ed. C. Jarlskog, Singapore World Scientific, 1989.

7. R. Aleksan et al., Phys. Lett. B316, 567 (1993).

8. C. Albajar et al.(UA1), Phys. Lett. B186, 247 (1987).

9. H. Albrecht et al. (ARGUS), Phys. Lett. B192, 245 (1987).

10. H.-G. Moser and A. Roussarie, Nucl. Instrum. Methods 384, 491 (1997).

11. SLD collab., SLAC-PUB-7228, SLAC-PUB-7229 and SLACPUB-7230, contrib. to 28th Int. Conf. on High Energy Physics, Warsaw, 1996;

J. Wittlin, PhD thesis, SLAC-R-582, 2001.

12. ALEPH collab., contrib. 596 to Int. Europhysics Conf. on High Energy Physics, Jerusalem, 1997.

13. J. Thom, PhD thesis, SLAC-R-585, 2002.

14. DELPHI collab., contrib. 303 to Int. Europhysics Conf. on High Energy Physics, Budapest, 2001. 
15. F. Abe et al.(CDF), Phys. Rev. Lett. 80, 2057 (1998), Phys. Rev. D59, 032001 (1999); Phys. Rev. D60, 051101 (1999); Phys. Rev. D60, 072003 (1999);

T. Affolder et al. (CDF), Phys. Rev. D60, 112004 (1999).

16. R. Barate et al. (ALEPH), Eur. Phys. J. C4, 367 (1998); Eur. Phys. J. C7, 553 (1999).

17. P. Abreu et al. (DELPHI), Eur. Phys. J. C16, 555 (2000); Eur. Phys. J. C18, 229 (2000).

18. M. Paulini, private communication.

19. B. Aubert et al.(BABAR), Phys. Rev. Lett. 87, 091801 (2001);

K. Abe et al. (Belle), Phys. Rev. Lett. 87, 091802 (2001).

20. D. Buskulic et al. (ALEPH), Z. Phys. C75, 397 (1997).

21. B. Aubert et al.(BABAR), hep-ex/0112044, SLACPUB-9061, to appear in Phys. Rev. Lett.; hep-ex/0112045, SLAC-PUB-9096, to appear in Phys. Rev. Lett.

22. K. Abe et al. (Belle), Phys. Rev. Lett. 86, 3228 (2001).

23. Y. Sakai, hep-ex/0202010, to appear in the proc. of the 5th KEK topical conference, 2001.

24. M. Paulini, Int. J. Mod. Phys. A14, 2791 (1999).

25. P. Abreu et al. (DELPHI), Z. Phys. C76, 579 (1997).

26. M. Acciarri et al. (L3), Eur. Phys. J. C5, 195 (1998).

27. G. Alexander et al. (OPAL), Z. Phys. C72, 377 (1996);

K. Ackerstaff et al.(OPAL), Z. Phys. C76, 401 (1997);

Z. Phys. C76, 417 (1997);

G. Abbiendi et al. (OPAL), Phys. Lett. B493, 266 (2000).

28. ALEPH, CDF, DELPHI, L3, OPAL, and SLD collab., "Combined results on $b$-hadron production rates and decay properties," CERN-EP/2001-050;

the combined results on $B$ mixing and $b$-hadron fractions included in the above paper or published in this Review have been obtained by the $B$ oscillations working group; see http://www.cern.ch/LEPBOSC/ for more information.

29. H. Albrecht et al. (ARGUS), Z. Phys. C55, 357 (1992); Phys. Lett. B324, 249 (1994).

30. J. Bartelt et al. (CLEO), Phys. Rev. Lett. 71, 1680 (1993).

31. B.H. Behrens et al. (CLEO), Phys. Lett. B490, 36 (2000).

32. D.E. Jaffe et al. (CLEO), Phys. Rev. Lett. 86, 5000 (2001).

33. F. Abe et al.(CDF), Phys. Rev. D55, 2546 (1997).

34. K. Ackerstaff et al. (OPAL), Z. Phys. C76, 401 (1997).

35. DELPHI collab., contrib. 449 to Int. Europhysics Conf. on High Energy Physics, Jerusalem, 1997. 
36. R. Barate et al. (ALEPH), Eur. Phys. J. C20, 431 (2001).

37. B. Aubert et al. (BABAR), hep-ex/0202041, SLACPUB-9149, submitted to Phys. Rev. Lett.

38. G. Abbiendi et al. (OPAL), Eur. Phys. J. C12, 609 (2000).

39. M. Beneke, G. Buchalla, and I. Dunietz, Phys. Lett. B393, 132 (1997);

I. Dunietz, Eur. Phys. J. C7, 197 (1999).

40. See the review on the CKM quark-mixing matrix by F.J. Gilman, K. Kleinknecht and B. Renk in this publication.

41. For a review of lattice QCD results with heavy quarks, see for example C. Bernard, Nucl. Phys. (Proc. Supp.) B94, 159 (2001).

42. A. Heister et al. (ALEPH), CERN/EP-2002-016, submitted to Eur. Phys. J. C.

43. F. Abe et al.(CDF), Phys. Rev. Lett. 82, 3576 (1999).

44. W. Adam et al. (DELPHI), Phys. Lett. B414, 382 (1997).

45. DELPHI collab., contrib. 304 to Int. Europhysics Conf. on High Energy Physics, Budapest, 2001.

46. G. Abbiendi et al. (OPAL), Eur. Phys. J. C11, 587 (1999); Eur. Phys. J. C19, 241 (2001).

47. SLD collab., SLAC-PUB-8568 and SLAC-PUB-8598, contrib. to 30th Int. Conf. on High Energy Physics, Osaka, 2000; C.-J.S. Lin, PhD thesis, SLAC-R-584, 2001.

48. D. Abbaneo, "Review of $B_{s}^{0}$ oscillations," to appear in the proc. of the 16th Rencontres de la Vallée d'Aoste, La Thuile, 2002.

49. Workshop on the CKM unitarity triangle, CERN, February 2002, http://www.cern.ch/ckm-workshop/, CERN yellow report in preparation.

50. K. Hartkorn and H.-G. Moser, Eur. Phys. J. C8, 381 (1999).

51. M. Acciarri et al. (L3), Phys. Lett. B438, 417 (1998).

52. F. Abe et al. (CDF), Phys. Rev. D59, 032004 (1999).

53. F. Abe et al. (CDF), Phys. Rev. D57, 5382 (1998).

54. R. Barate et al. (ALEPH), Phys. Lett. B486, 286 (2000).

55. See for example M. Beneke, G. Buchalla, and I. Dunietz, Phys. Rev. D54, 4419 (1996).

56. See the review on production and decay of $b$-hadrons by L. Gibbons and K. Honscheid in this publication.

57. D. Buskulic et al. (ALEPH), Phys. Lett. B377, 205 (1996); K. Ackerstaff et al. (OPAL), Phys. Lett. B426, 161 (1998).

58. P. Abreu et al. (DELPHI), Phys. Lett. B289, 199 (1992); P.D. Acton et al. (OPAL), Phys. Lett. B295, 357 (1992); 
D. Buskulic et al. (ALEPH), Phys. Lett. B361, 221 (1995).

59. P. Abreu et al. (DELPHI), Z. Phys. C68, 375 (1995);

R. Barate et al. (ALEPH), Eur. Phys. J. C2, 197 (1998).

60. P. Abreu et al. (DELPHI), Z. Phys. C68, 541 (1995);

D. Buskulic et al. (ALEPH), Phys. Lett. B384, 449 (1996).

61. R. Barate et al. (ALEPH), Eur. Phys. J. C5, 205 (1998).

62. DELPHI collab., contrib. 5_515 to Int. Europhysics Conf. on High Energy Physics, Tampere, 1999.

63. F. Abe et al.(CDF), Phys. Rev. D60, 092005 (1999);

T. Affolder et al. (CDF), Phys. Rev. Lett. 84, 1663 (2000).

64. K. Anikeev et al., " $B$ physics at the Tevatron: Run II and beyond," hep-ph/0201071, FERMILAB-PUB-01/97. 